\title{
Gasless transaxillary endoscopic thyroidectomy for unilateral low- risk thyroid cancer: Li's six-step method
}

\author{
Yuqiu Zhou ${ }^{1 \#}$, Yongcong Cai ${ }^{1 \#}$, Ronghao Sun ${ }^{1}$, Chunyan Shui ${ }^{1}$, Yudong Ning ${ }^{1}$, Jian Jiang ${ }^{1}$, Wei Wang ${ }^{1}$, \\ Jianfeng Sheng ${ }^{2}$, Zhenhua Jiang ${ }^{3}$, Zhengqi Tang ${ }^{4}$, Wen Tian ${ }^{5}$, Chuanming Zheng ${ }^{6}$, Minghua Ge ${ }^{6}$, \\ Chao $\mathrm{Li}^{1}$
}

${ }^{1}$ Department of Head and Neck Surgery, Sichuan Cancer Hospital, Sichuan Cancer Institute, Sichuan Cancer Prevention and Treatment Center, Cancer Hospital of University of Electronic Science and Technology School of Medicine, Chengdu, China; ${ }^{2}$ Department of Thyroid, Head, Neck and Maxillofacial Surgery, The Third People's Hospital of Mianyang, Sichuan Mental Health Center, Mianyang, China; ${ }^{3}$ Department of Head and Neck Surgery, Central Hospital of Mianyang City, Mianyang, China; ${ }^{4}$ Department of Otolaryngology Head and Neck Surgery, Zigong Third People's Hospital, Zigong, China; ${ }^{5}$ Department of General Surgery, Chinese PLA General Hospital, Beijing, China; ${ }^{6}$ Department of Head and Neck Surgery, Center of Otolaryngology-head and Neck Surgery, Zhejiang Provincial People's Hospital, People's Hospital of Hangzhou Medical College, Hangzhou, China

\#These authors contributed equally to this work.

Correspondence to: Chao Li. Department of Head and Neck Surgery, Sichuan Cancer Hospital, Sichuan Cancer Institute, Sichuan Cancer Prevention and Treatment Center, Cancer Hospital of University of Electronic Science and Technology School of Medicine, Chengdu 610041, China. Email: headneck@qq.com.

\begin{abstract}
The past decade has witnessed rapid advances in gasless transaxillary endoscopic thyroidectomy (GTET) for thyroid cancer, which has become a reliable procedure with good therapeutic effectiveness, aesthetic benefits, and safety. This procedure has been widely promoted in some Asian countries; however, few studies have described the specific surgical steps for unilateral low-risk thyroid cancer. Based on a review of the literature and our own clinical experience, we introduce in detail the surgical procedure of GTET for the unilateral low-risk thyroid cancer, briefly summarized into Li's 6 steps: surgical position and incision design; creation of surgical cavities; dissection of the superior pole of the thyroid and its vessels, and identification and protection of superior laryngeal nerve; identification and protection of the superior parathyroid gland, and identification of the inferior parathyroid gland; identification and protection of the recurrent laryngeal nerve and the inferior parathyroid gland, and central neck dissection; and processing of the suspensory ligaments of thyroid gland and en bloc resection of the tumor. The six-step approach is simple to learn. The lymph nodes are dissected first, followed by resection of the primary lesion and protect important structures, which meets the principles of radical tumor treatment. It is hoped that the proposed Li's six-step method can promote the standardized, safe, and wide application in treating early thyroid cancer.
\end{abstract}

Keywords: Thyroidectomy; thyroid cancer; endoscopy; transaxillary thyroidectomy; gasless

Submitted Jan 19, 2021. Accepted for publication May 19, 2021.

doi: $10.21037 / g s-21-257$

View this article at: http://dx.doi.org/10.21037/gs-21-257

\section{Introduction}

Gasless transaxillary endoscopic thyroidectomy (GTET) was first described by Yoon et al. in 2006 (1), and the past decade has witnessed the rapid advancement in this procedure, which can now achieve good clinical efficacy and provide cosmetic benefits. This procedure is performed through an incision within the natural wrinkle line in the axillary natural fold to avoid scarring the neck; also, the cavity is built through the natural gap between the pectoralis major muscle and the neck, which avoids damage to normal structures and can prevent postoperative sensory 

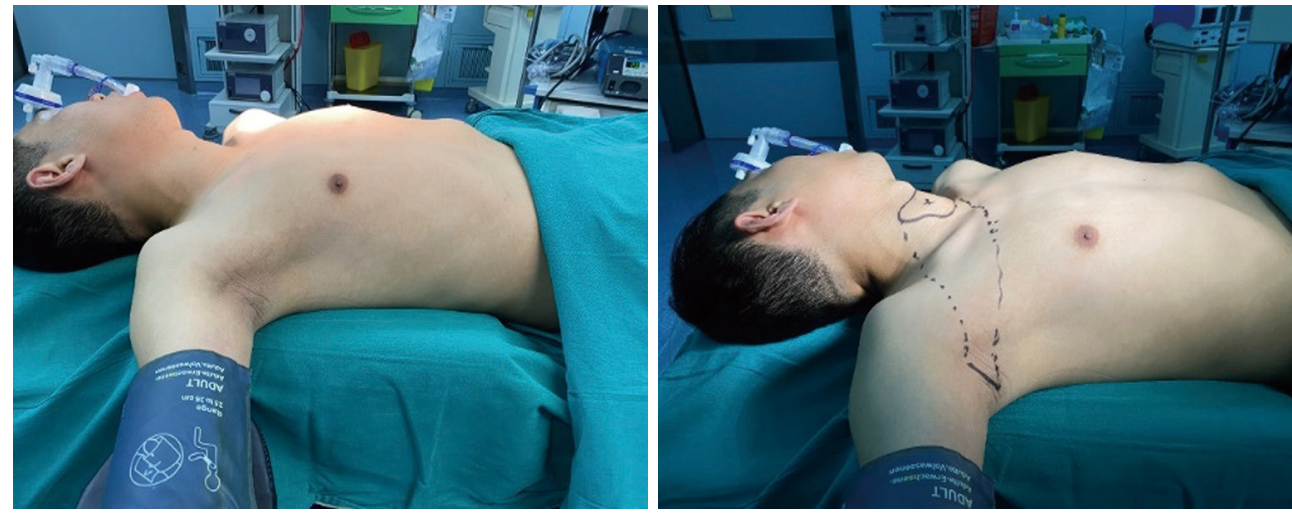

Figure 1 Surgical position and incision.

abnormalities in the anterior neck and skin synkinesis during swallowing. The procedure is safe and does not require $\mathrm{CO}_{2}$ gas insufflation, which reduces gas-related complications while maintaining a clear operative field. As the axillary incision is larger than that of other endoscopic incisions, this approach also allows for the removal of relatively large thyroid tumor. This endoscopic approach is relatively easy to perform, has a stable cavity-forming and avoids any visible, external scars to the neck. At present, this procedure is being applied in centers across the world, and the techniques related to thyroid lobectomy have been summarized (2). However, few studies have described the specific surgical steps for the radical treatment of thyroid cancer. Based on the previous literature $(3,4)$ and the clinical practice of the Head and Neck Surgery Team in Sichuan Cancer Hospital, we here summarize the surgical steps (also see Video 1 for details), operation techniques, and precautions in GTET for the radical treatment of unilateral low-risk thyroid cancer. Among these steps, lymph nodes are dissected first, followed by resection of the primary lesion and protect important structures, which meets the principles of radical tumor treatment. The procedure, called Li's six-step approach, is described here in an attempt to promote its safety and standardized application in clinical settings. This six-step approach is simple and feasible and can be widely adopted by more centers.

\section{Surgical steps, operating techniques, and precautions}

This report has been approved by the ethics committee of Sichuan Cancer Hospital (approval No. SCCHEC-03-2019-009), with all patients signing the informed consent forms. All procedures performed in this study involving human participants were in accordance with the Declaration of Helsinki (as revised in 2013).

\section{Step 1: body position and incision design}

Under general anesthesia, the patient is placed in supine position on a pad positioner, with the head tilted about $45^{\circ}$ to the healthy side. The affected upper limb is naturally abducted $90-180^{\circ}$. If the clavicle at the affected side is higher than the thyroid isthmus under direct visualization, the angle of the upper limb abduction is appropriately adjusted to higher than $90^{\circ}$ until the clavicle is inferiorly displaced below the level of the thyroid isthmus. After the axilla at the affected side is exposed (Figure 1), an incision is made under the natural folds in the armpit. A trocar is placed in the axillary incision about two fingers away from the breast side and slightly below the anterior axillary line. After disinfection and draping are routinely performed in the surgical field, the surgical incision is made according to the preoperative design. Cut open subcutaneous fatty layer until the upper lateral edge of the pectoralis major muscle is exposed.

Operating skills and precautions: (I) the proper axillary fold should be selected, as it affects the aesthetic outcome. The incision is about 4-6 cm long and should not exceed the anterior axillary line. An operation along the anterior axillary line is more convenient but is associated with poorer aesthetic outcome. (II) The trocar can be inserted when the flap has been fixed at the beginning of suspension hook placement. The insertion site can also be adjusted according to the actual situation and personal preferences. The farther the site is away from the axillary incision, the less obvious 
the "chopstick effect" (i.e., the interference among multiple instruments) is; however, the location of the puncture point may be associated with poorer aesthetic outcome: if the puncture site is closer to the incision, the chopstick effect will become obvious. Meanwhile, the postoperative puncture site has a less obvious effect. Nevertheless, the puncture point should be kept above the pectoralis major muscle to avoid the obstruction of the instrumentation by the muscle.

\section{Step 2: creation of surgical cavities}

The creation of surgical cavities mainly follows the threestep method, which involves lifting the surface skin of the pectoralis major muscle to create the chest wall cavity, lifting the sternocleidomastoid muscle or its sternal head to create the carotid sheath cavity, and lifting the strap muscle to create the thyroid cavity. The cavity is separated from the 2 ends of the incision to suprasternal fossa (lower border) and pyramidal lobe of thyroid (upper border). The specific steps are as follows: with the assistance of a retractor, the gap between the muscle and subcutaneous fat is separated with a long-headed electric knife (Figure 2A). The integrity of the membrane of the pectoralis major muscle is maintained during the division. When the electric knife is unable to continue the division, it is replaced with a suspension hook before the division is performed under endoscopy (Figure 2B). The subcutaneous tunnel is separated along the demarcated area by using an electrocantery or ultrasonic scalpel (Figure 2C). The supraclavicular nerve, a branch of the cervical plexus nerve, should be carefully protected when the separation crosses the clavicle (Figure 2D) to reduce postoperative numbness in the supraclavicular region. The sternocleidomastoid muscle bundle will be exposed when the separation crosses the clavicle, and the posterior border of the sternocleidomastoid muscle can be visible with a little separation. The yellowishwhite natural gap between the sternal and clavicular heads of the sternocleidomastoid muscle (Figure 2E) or the posterior edge of the muscle (Figure $2 F$ ) is exposed naturally or after retraction with an endoscopic device. The fascial tissues are separated along the natural gap, and the sternal head (Figure 2G) or the posterior edge of the muscle is lifted by moving the suspension hook (Figure $2 H$ ). After the cavity is created, the omohyoid muscle (Figure $2 I$ ) and the carotid sheath are exposed and then dissect the omohyoid muscle. The omohyoid muscle is an important anatomical landmark, with the carotid sheath and the strap muscle in its deep surface. A "muscular triangle" is formed at the junction between the lateral side of the strap muscle and the omohyoid muscle (Figure 27), and the thyroid gland is exposed by separation along the gap between the lateral side of the strap muscle and the carotid sheath (Figure $2 K$ ). The superior border is the superior pole of the thyroid gland, the inferior border is the suprasternal fossa, the medial border slightly crosses the isthmus of the thyroid gland to the contralateral gland, and the lateral border is the medial edge of the carotid artery. Whether the omohyoid muscle is preserved or not depends on the operator's proficiency and preference or depends on the exposure of the surgical field. The lateral border of the strap muscle is identified through the muscular triangle. The deep side of the strap muscle is separated for straight exposure of the deep side of the glandular fascia, and the retractor is advanced to lift the strap muscle to complete the third step of cavity creation. The 2 sides of the sternocleidomastoid muscle are appropriately loosened and lowered according to the visibility of the intraoperative field.

Operating skills and precautions: (I) when the separation passes across the clavicle, there is no the pectoralis major muscle in the field of view, but adipose and fascial connective tissues. Excessive separation towards the deep side may cause brachial plexus injuries and ipsilateral upper limb lymphedema (4). The separation gap can be exposed by increasing the pulling force of the retractor during separation. (II) The gap in the sternocleidomastoid muscle may be not obvious in some patients, it is necessary to carefully distinguish the distribution of muscle bundles, or to search for in by using separation forceps. For some patients in whom exposing the gap is difficult, the gap at the posterior edge of the sternocleidomastoid muscle has a relatively fixed anatomy, and therefore the posterior approach can be chosen. (III) During separation of the gap in the sternocleidomastoid muscle, the distance between the 2 sides of the separation can be slightly larger than the width of the retractor. When the separation proceeds to the cephalad side, any injury to the external jugular vein should be avoided, with active avoidance being the best option. (IV) The strap muscle is composed of the sternohyoid muscle and the sternothyroid muscle. Both muscles can be lifted together for separation, which avoids further bleeding and trauma caused by separating them one by one. In addition, excessive separation of the strap muscle should be avoided; the separation is sufficient if the retractor can be inserted, which allows for the traction of the gland by the connection between the gland and the muscle and facilitates traction of 

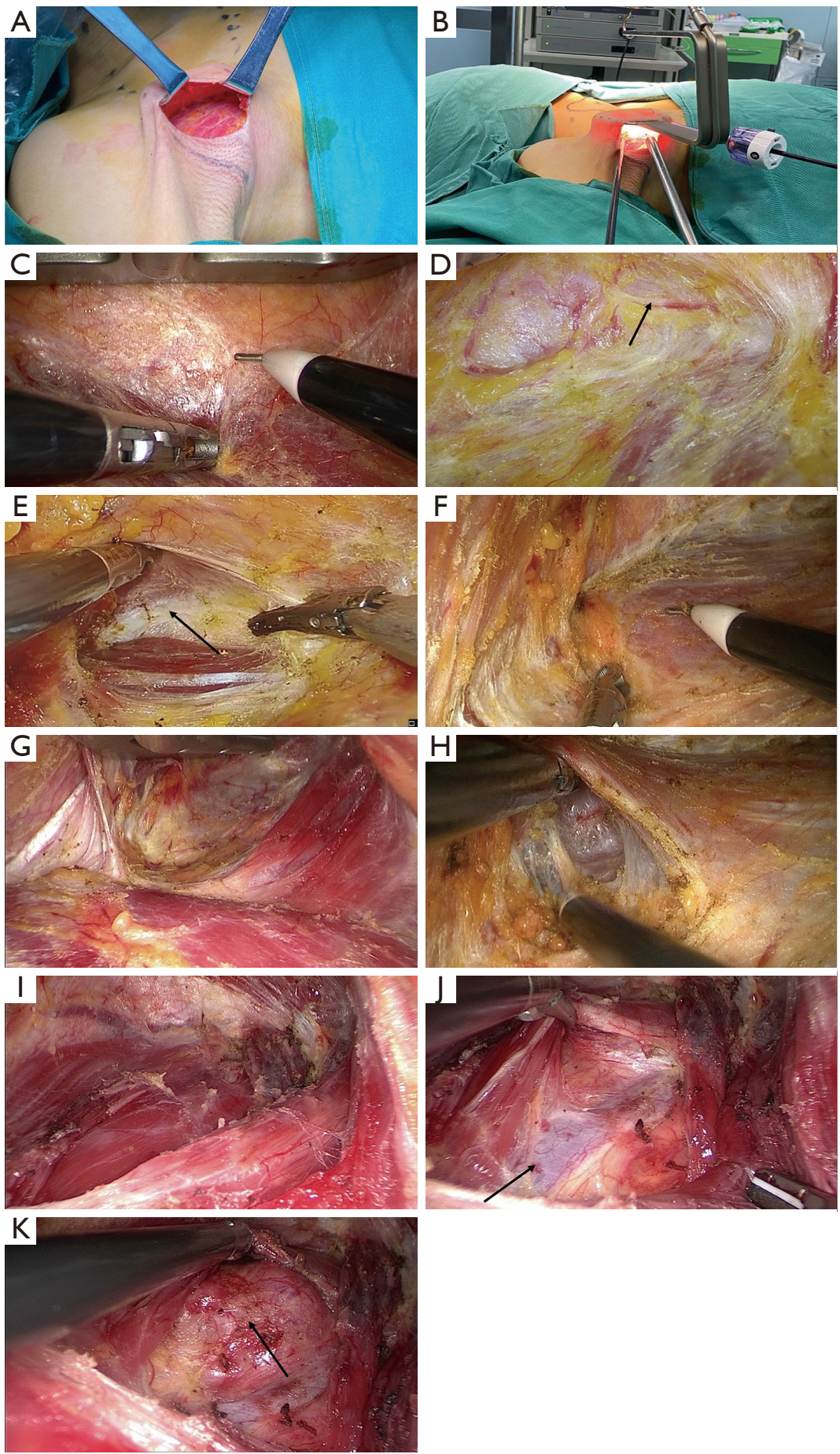

Figure 2 Creation of a surgical cavity. (A) Exposure of the lateral edge of the pectoralis major muscle. (B) Placement of operating instruments. (C) Separation of the pectoralis major myocutaneous flap. (D) Protection of the supraclavicular cutaneous nerve (arrow shows the cutaneous nerve). (E) Identification of the sternocleidomastoid muscle gap (arrow shows the muscle gap). (F) Identification of the posterior edge of sternocleidomastoid muscle. $(\mathrm{G})$ Separation of the sternocleidomastoid muscle gap. (H) Separation of the posterior edge of sternocleidomastoid muscle. (I) Dissection of the omohyoid muscle (arrow shows the omohyoid muscle). (J) Exposure of the "muscular triangle" at the junction between the lateral side of strap muscle and the omohyoid muscle (arrow shows the internal jugular vein). (K) Traction of the strap muscle to expose the thyroid (the arrow shows the thyroid). 

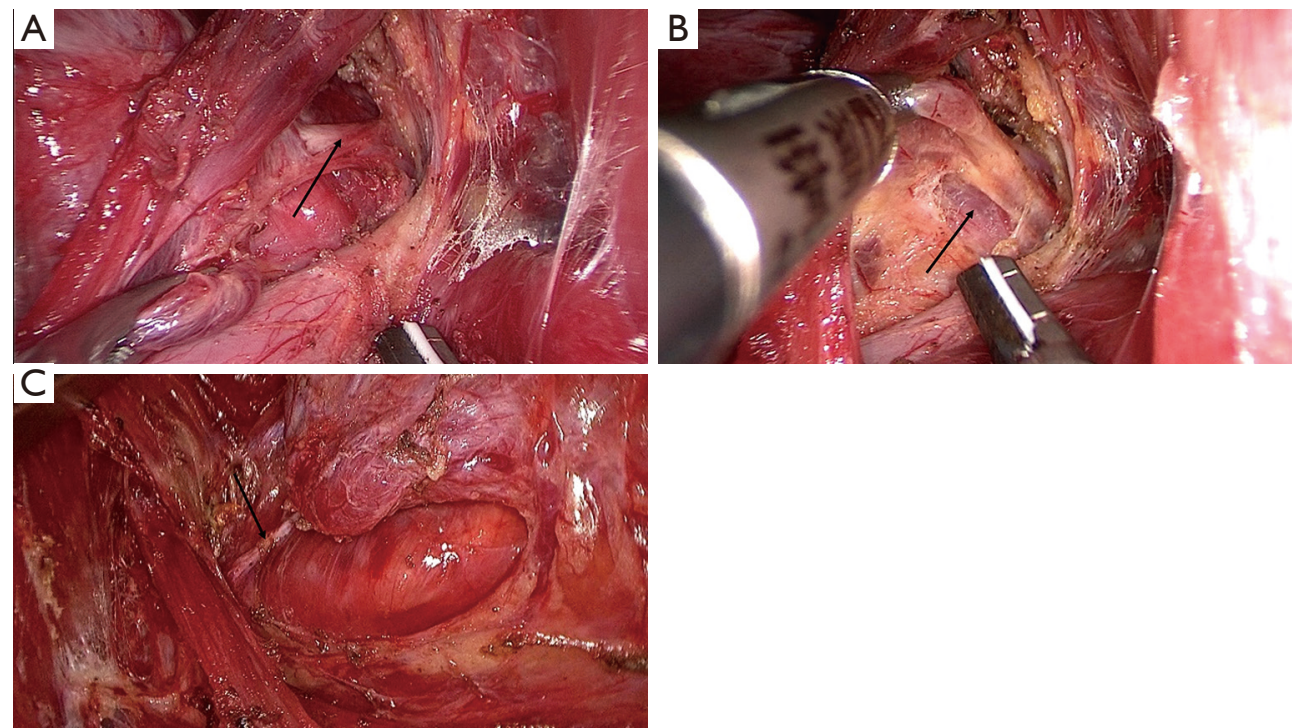

Figure 3 Dissection of the superior pole of the thyroid and its vessels and identification and protection of the superior parathyroid gland and superior laryngeal nerve. (A) Skeletonization of the superior thyroid vessels (arrows). (B) Exposure of the cricothyroid space (arrow). (C) Identification and protection of the superior laryngeal nerve (arrow).

the recurrent laryngeal nerve to the surface of the trachea, exposure of deep side of the recurrent laryngeal nerve of the tracheoesophageal groove and central neck dissection. (V) The external jugular vein should be carefully protected when the posterior edge of the sternocleidomastoid approach is adopted.

\section{Step 3: dissection of the superior pole of the thyroid and its vessels, and identification and protection of the superior laryngeal nerve}

The superior region of the gland is finely dissected to separate the true and false peritonea, for protecting the superior parathyroid glands, and their adjacent fatty connective tissues. The superior polar thyroid gland is separated and completely exposed, and the superior thyroid vessels are skeletonized (Figure $3 A$ ) while the cricothyroid space is separated (Figure 3B). The ultrasonic scalpel at multiple points to reduce the risk of bleeding and avoid damage to the superior laryngeal nerve. It is helpful to reduce bleeding during subsequent surgery to process the superior thyroid vessels. The superior laryngeal nerve can also be identified, dissected, and protected first (Figure 3C) before coagulation of the superior thyroid vessels. Intraoperative nerve monitoring system can be used to assist in identifying and protecting the superior laryngeal nerve (5).

Operating skills and precautions: (I) The separation close to the thyroid capsule should be ensured, and the superior parathyroid gland and adjacent connective tissues should be actively identified and protected. (II) Skeletonization of the superior thyroid vessels can reduce injuries to deep important structures such as the cricothyroid muscle and the superior laryngeal nerve during the using of energy devices. (III) For patients with high position of the superior thyroid vessels and large thyroid gland, the exposure of the superior pole vessels may be difficult; in such cases, a small amount of the strap muscle can be further separated towards the head (with the muscle being cut off if necessary). The superior thyroid pole is pulled downwards to facilitate the skeletonization and coagulation of the superior thyroid vessels. (IV) During the processing of the superior pole thyroid gland, the omohyoid muscle can also be cut off if it covers surgical field.

\section{Step 4: identification and protection of the superior parathyroid gland, and identification of the inferior parathyroid gland}

The superior parathyroid glands have relatively fixed locations, with $80-85 \%$ of them in a circular area with a radius of $1 \mathrm{~cm}$ centered on the inferior angle of the thyroid 

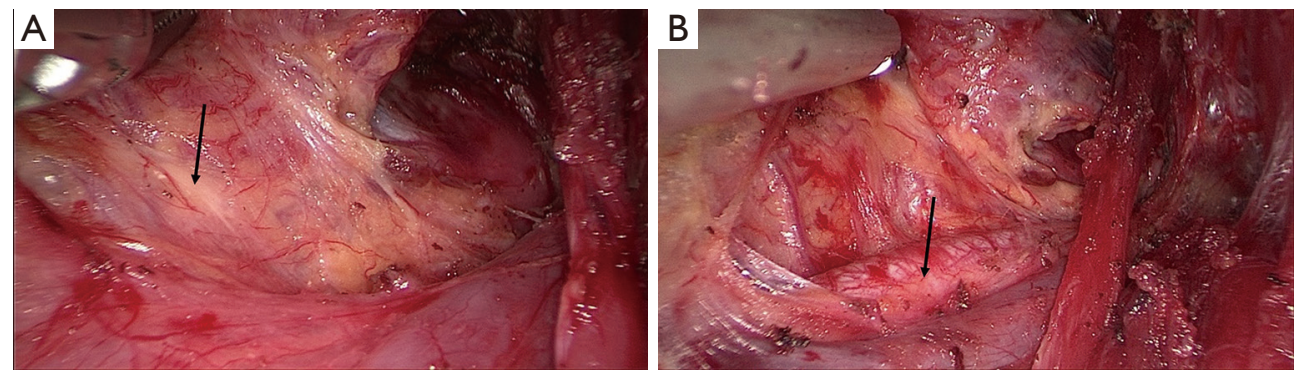

Figure 4 Identification and protection of the superior parathyroid gland and identification of the inferior parathyroid gland. (A) Identification and protection of the superior parathyroid glands (the arrows show the superior and inferior parathyroid glands). (B) Dissect the medial edge of the carotid artery and expose the external border of the central neck dissection (the arrow shows the carotid artery).

cartilage (or $1 \mathrm{~cm}$ above the intersection of the recurrent laryngeal nerve and the inferior thyroid artery) (6). The superior thyroid gland is lifted outwards and upwards, then the superior parathyroid glands and their surrounding vascular networks are exposed and protected by using a "decapping" method. (Figure 4A) The locations of the inferior parathyroid glands are highly variable, with most glands (approximately $80 \%$ ) located in the area between the lower pole of the thyroid gland and the thymus. Thus, the inferior parathyroid glands must be identified before lymph node dissection. Coagulate the middle thyroid vein, dissect the medial edge of the carotid artery (Figure $4 B$ ) and expose the external border of the central neck dissection. As en bloc resection of the tumor is required during the surgery, central neck dissection is performed throughout the procedure.

Operating skills and precautions: the superior parathyroid has a relatively fixed position and should be preserved in situ, if possible. The gland and the tissue in the central neck could be pulled upward by a suspension hook, so that the inferior parathyroid gland can be searched for in the middle and upper portions of the visual field.

\section{Step 5: exposure, dissection, and protection of the recurrent laryngeal nerve and the inferior parathyroid gland, and central neck dissection}

Blunt separation in the middle and lower areas of the tracheoesophageal groove is performed to expose the inferior thyroid artery and the recurrent laryngeal nerve (the latter is vertically across the deep side of the recurrent laryngeal nerve) (Figure 5A). The surface tissue is bluntly separated along the route of the recurrent laryngeal nerve towards the nerve entry into the larynx, and the nerve is dissected and protected. Meanwhile, the inferior thyroid artery is coagulated at multiple points (Figure $5 B$ ).

The boundary of lymph node dissection in central neck is determined. The lateral boundary is the medial edge of the carotid artery, the lower boundary is the suprasternal fossa, the deep site is the muscular layer of the esophagus and the prevertebral fascia, and the midline is the outer edge of the healthy side of the trachea. Along the above boundary, the deep or lateral lymph nodes of the recurrent laryngeal nerve were dissected first, then lose the recurrent laryngeal nerve, finally dissect superficial or medial lymph nodes of the recurrent laryngeal nerve and that of pretracheal, and lift these lymph nodes. (Figure 5C). The inferior artery of thyroid and its surrounding veins are coagulated as close as possible to the gland to ensure the blood supply to the parathyroid glands (Figure 5D). The lymph nodes at the thoracic inlet are more difficult to expose, resulting in a severe chopstick effect. Thus, the positions of the endoscopic instruments and laparoscope need to be adjusted and changed constantly. The thymus should be protected during the dissection of the pretracheal lymph nodes (Figure 5E) to prevent the misresection of the heterotopic parathyroid gland or damage of the blood supply of the inferior parathyroid gland $(7,8)$. Attention should also be paid to the occult lymph nodes beneath the thymus. Typically, the location of the inferior parathyroid glands is variable and more difficult to preserve. Auto transplantation should be adopted decisively for those parathyroid glands that cannot be preserved in situ, have stable blood supply or misresction. For parathyroid glands that cannot be preserved in situ, have no stable blood supply. The free glands can be placed in the pectoralis major muscle near the incision and marked with non-absorbable sutures $(6,9)$. Auxiliary intraoperative parathyroid identification and 

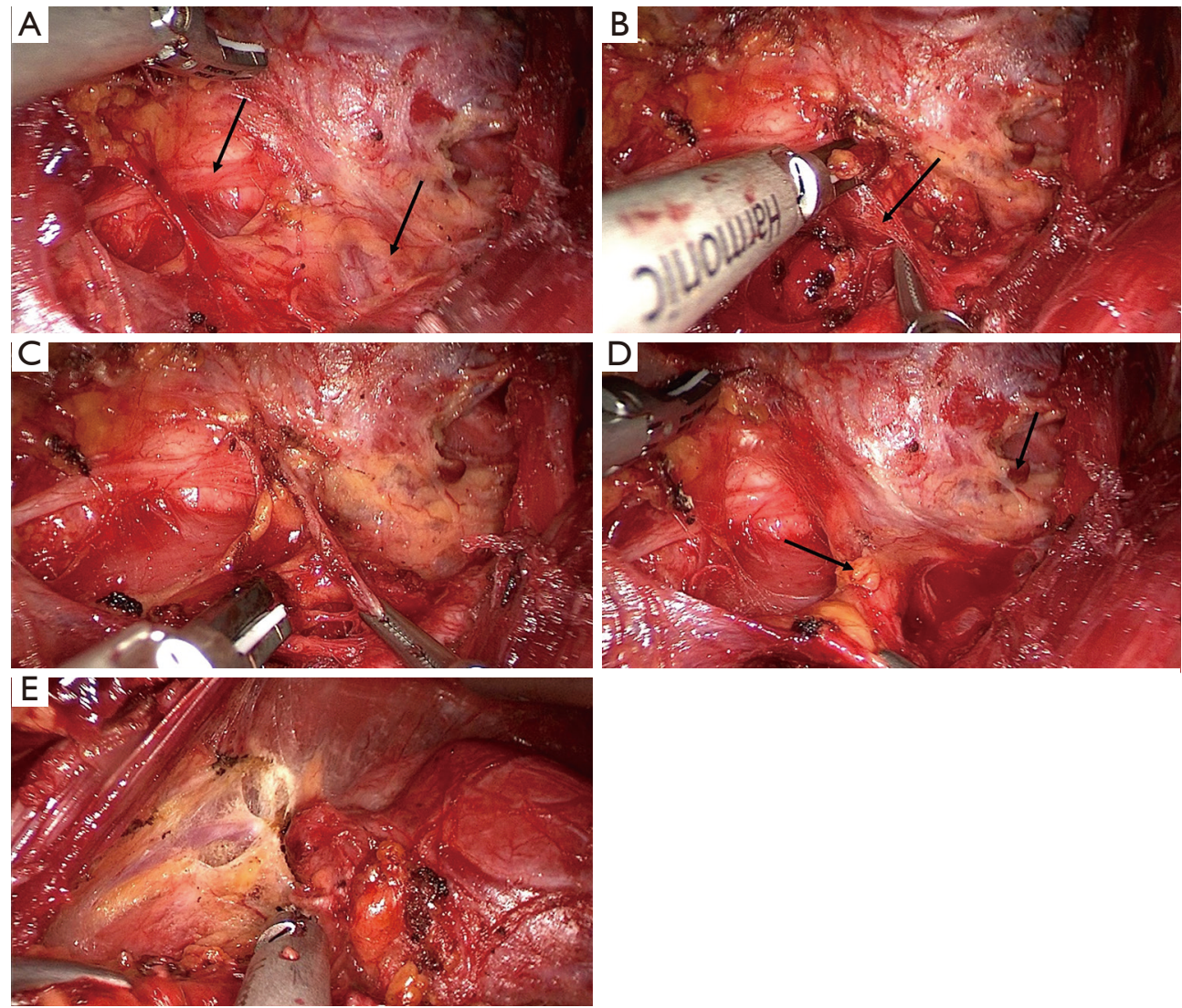

Figure 5 Exposure, dissection, and protection of the recurrent laryngeal nerve and the inferior parathyroid gland and central neck dissection. (A) Dissection and protection of the recurrent laryngeal nerve (the arrows show the recurrent laryngeal nerve and inferior thyroid artery). (B) Processing of the inferior thyroid artery (arrow). (C) Lymph node dissection in the tracheoesophageal groove. (D) Identification and protection of the superior and inferior parathyroid glands (arrows show the superior and inferior parathyroid glands). (E) Dissection of the pretracheal lymph nodes.

protection techniques may be used to facilitate parathyroid identification and protection (10).

Operating skills and precautions: (I) intraoperative nerve monitoring system can also be used to assist in the identification and protection of the recurrent laryngeal nerve (11). (II) The working head of the energy instruments should be kept away from the recurrent laryngeal nerve when dissecting the perineural tissues, with a safe distance of at least 3-5 mm. Selection of a low-power mode can increase the safety. In addition, a small strip of wet gauze strip should be used, if possible, to isolate the ultrasonic scalpel from the important adjacent structures (12). (III) Lymph node dissection at the thoracic inlet is challenging. Therefore, preoperative computed tomography (CT) and other imaging assessments in this area are particularly important. The position of the recurrent laryngeal nerve should be carefully observed, and any accidental injury to the nerve should be avoided. The nerve and adjacent lymph nodes are lifted in this area, and the lymph nodes must be dissected before the nerve is release. Because once the nerve is loosed, the structures of this area will descend resulting some areas may become blind. Energy instruments can be more used at low-power mode to ensure adequate coagulation of small vessels. (IV) The coagulated end of the inferior thyroid artery should be located in the surgical field of view to facilitate the procession of bleeding. (V) In some cases, the thyroid veins are distributed in the deep side of the pretracheal tissue, and energy instruments at low-power mode may be applied to coagulate these vessels during lymph node dissection. 

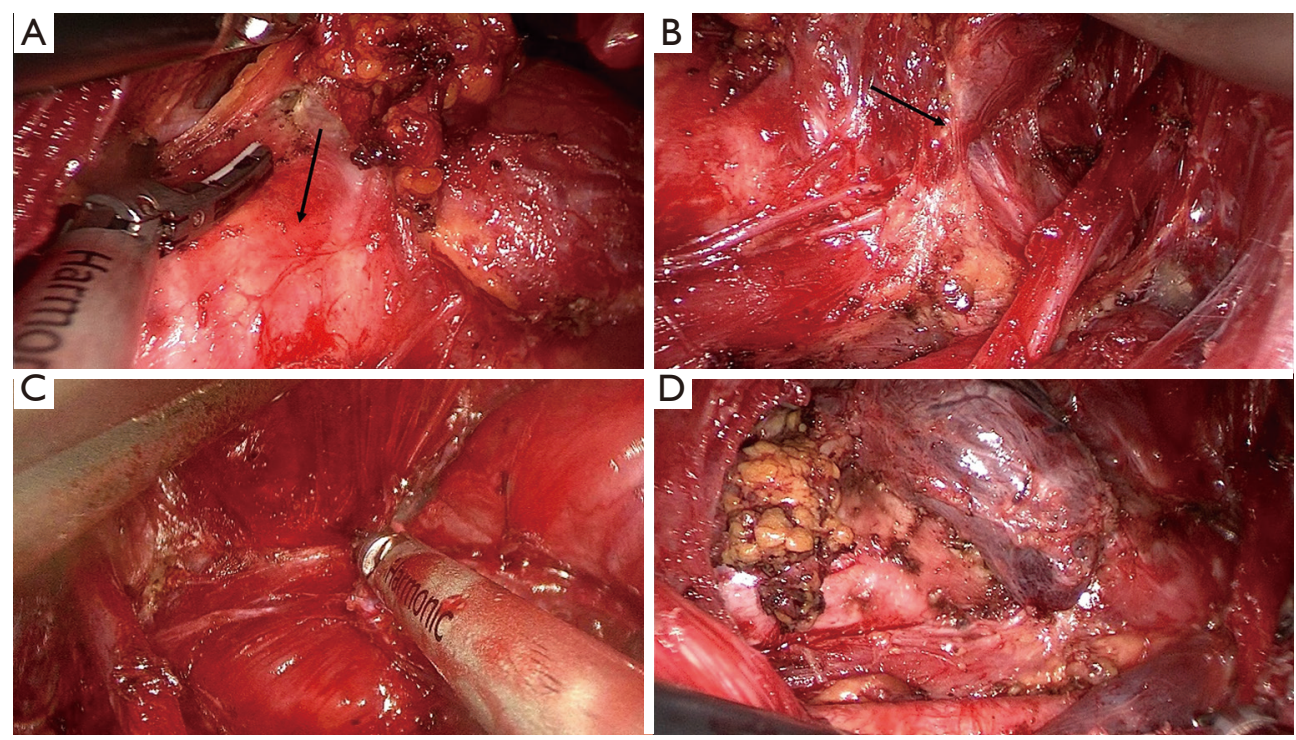

Figure 6 Processing of the suspensory ligaments of thyroid gland and en bloc resection of the tumor. (A) Processing of the inferior pole and isthmus of the thyroid (arrow shows the trachea). (B) Processing of the suspensory ligament of thyroid gland (arrow). (C) Processing of the pyramidal lobe and the prelaryngeal lymph nodes. (D) En bloc resection of the primary tumor and central lymph nodes.

\section{Step 6: processing of the suspensory ligament of thyroid gland and en bloc resection of the tumor}

After above steps, the gland is mostly mobilized, only the thyroid isthmus (Figure 6A), the suspensory ligament (Figure $6 B$ ), the pyramidal lobe, and the prelaryngeal lymph nodes (Figure $6 C$ ) remain to be processed. For the processing of the suspensory ligaments of thyroid gland, bipolar electrocoagulation followed by cutting can be considered to minimize thermal injury and bleeding and enable the complete resection of the glands. If an energy instrument is used, the suspensory ligament should be dissected in a single attempt, as multiple attempts may result in residual gland. Wet gauze strip is used to shield the RLN laryngeal entry point to reduce thermal injury. If the tissue in the suspensory ligament is dense, an electrocantery or bipolar coagulation can be used to separate and dissect the ligament layer by layer. The excised tumor and lymph nodes (Figure 6D) are removed as a whole from the axillary incision.

Operating skills and precautions: (I) there are many tiny vessels and dense tissues at the RLN laryngeal entry point, and bleeding caused by excessive separation with endoscopic instruments should be avoided. Wet gauze strip can be used to push the RLN downwards to expose the gap. (II) In some patients, a small number of glands may be preserved at the suspensory ligament as appropriate to ensure postoperative voice function and quality of life. (III) Despite the large size of axillary cavity, direct removal of the specimens from the axillary cavity should be avoided, and transport by using a specimen bag can reduce the risk of thyroid or tumor implantation (13-15).

The surgical cavity is irrigated. Adequate hemostasis is required, as sternocleidomastoid muscle, clavicle, and supraclavicular fossa are easy to ooze blood. A drainage tube is placed through the axillary incision. The axillary incision is sutured layer by layer. Finally, bandage with pressure applying in the axillary, supraclavicular, and cervical areas.

\section{Discussion}

In this article we summarize the operation skills and precautions of GTET for the radical treatment of thyroid cancer. For further understanding and mastering, we also provide cartoon figures (Figure 7). The advantages of our procedure we conclude are as follow: (I) the three-step cavity creation method is used to expose and create a chest wall cavity, a carotid sheath cavity, and finally a thyroid cavity by suspending each structure using a retractor. A fascial layer approach in the gap is used during the creation of the first 2 cavities to avoid muscle injuries. During the creation of the third cavity, the muscle triangle should be identified, at which the strap muscle is lifted to create the thyroid cavity. 

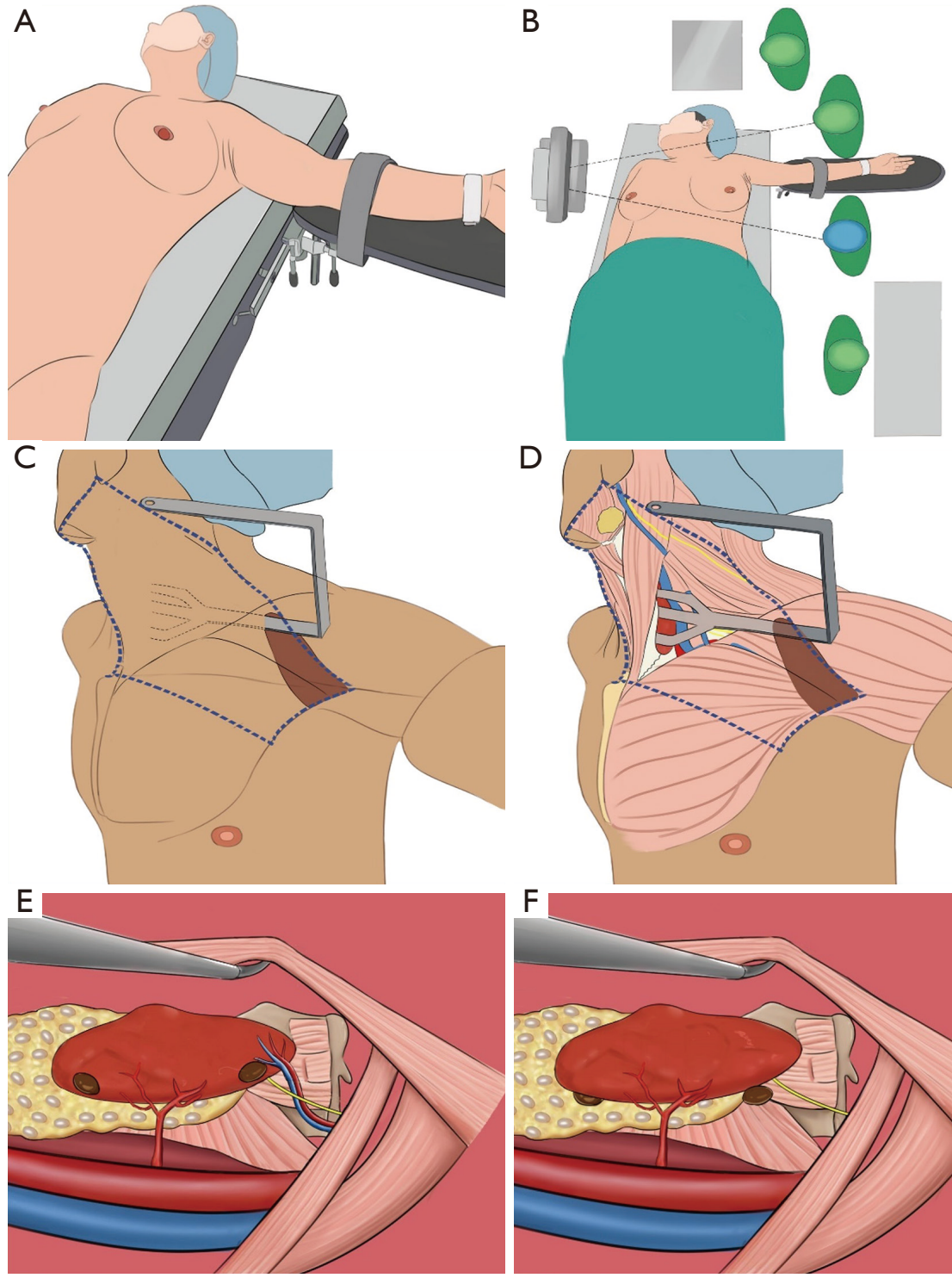

$\mathrm{D}$
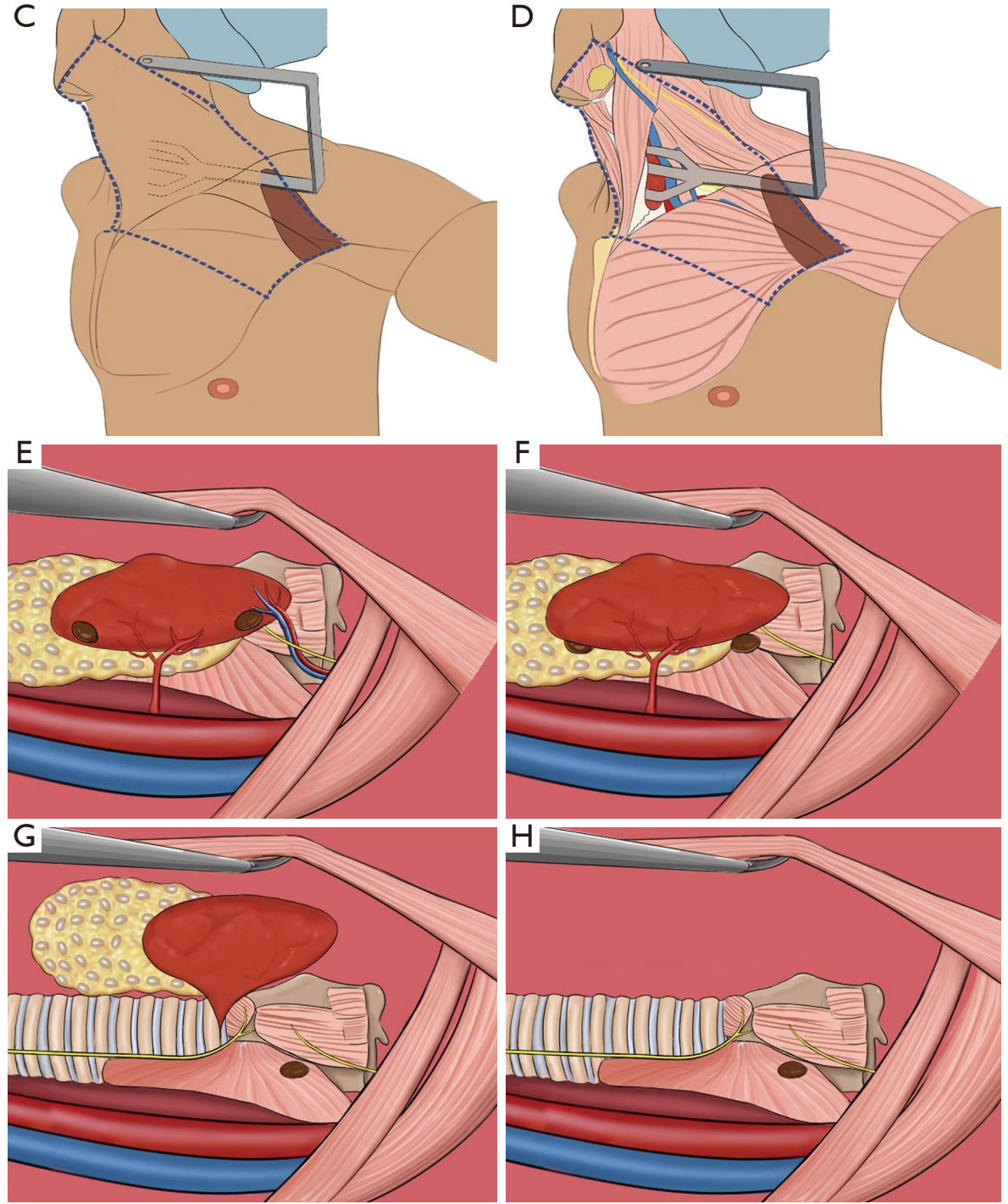

Figure 7 The cartoon figures for Methods of gasless transaxillary endoscopic thyroidectomy for unilateral low-risk thyroid cancer. (A) Body position. (B) Operation room setup: team. (C,D) Creation of surgical cavities. (E) Identification of the superior thyroid vessels, the superior laryngeal nerve and the parathyroid glands. (F) Processing the superior thyroid vessels and protecting the superior laryngeal nerve and the parathyroid glands. (G) Dissection and protection of the recurrent laryngeal nerve and processing of the suspensory ligaments of thyroid gland. $(\mathrm{H})$ The structures after en bloc resection of the primary tumor and central lymph nodes. 
(II) After the cavities creation, give priority to processing superior and in inferior thyroid artery, which facilitates bleeding reducing and operating field clearance. (III) Important structures such as the RLN, superior and inferior parathyroid glands are actively exposed and protected, which facilitates radical resection and reduction of postoperative complications. The endoscope's magnification permits more accurate and delicate manipulation. With the use of the transaxillary approach, it is easier to identify, dissect, and protect important structures, especially the superior and inferior polar vessels of the thyroid, the RLN, and the superior and inferior parathyroid glands. (IV) After exposure of important structures, give priority to central neck dissection in the sequence of bottom to top and inward to outward. Dissect interior border of the carotid artery to superior border of the innominate artery to determine boundary of neck dissection. During the processing of the area near the RLN, separation using a "cold" device is preferred to minimize stretch and/or thermal damage. The glands are completely mobilized, followed by the processing of the suspensory ligament area. Finally, en bloc resection of the primary lesion and the central lymph nodes is performed, which follows the principles of tumor-free operation. However, the best indication of our method is cT1-2N0 patients because a blind spot may exist in the visual field when performing lymph node dissection in the posterior sternal region (16). Preoperative evaluation of the central region using contrast-enhanced CT and/or color Doppler ultrasound is recommended, especially for the substernal area. With the accumulation of experience and operation proficiency of surgeons. This method is recommended for some cT3aN1a patients without metastatic lymph nodes in substernal area. In addition, this method is more difficult in processing contralateral glands and in dealing with central lymph nodes on the same side (17); therefore, it is more feasible for unilateral low-risk thyroid cancer. It is hoped that the summary of the surgical steps and the discussion of the precautions in each step will further inform and promote the application of this technique in more clinical settings.

\section{Acknowledgments}

Funding: None.

\section{Footnote}

Conflicts of Interest: All authors have completed the ICMJE uniform disclosure form (available at http://dx.doi. org/10.21037/gs-21-257). The authors have no conflicts of interest to declare.

Ethical Statement: The authors are accountable for all aspects of the work in ensuring that questions related to the accuracy or integrity of any part of the work are appropriately investigated and resolved. This report has been approved by the ethics committee of Sichuan Cancer Hospital (approval No. SCCHEC-03-2019-009), with all patients signing the informed consent forms. All procedures performed in this study involving human participants were in accordance with the Declaration of Helsinki (as revised in 2013).

Open Access Statement: This is an Open Access article distributed in accordance with the Creative Commons Attribution-NonCommercial-NoDerivs 4.0 International License (CC BY-NC-ND 4.0), which permits the noncommercial replication and distribution of the article with the strict proviso that no changes or edits are made and the original work is properly cited (including links to both the formal publication through the relevant DOI and the license). See: https://creativecommons.org/licenses/by-nc-nd/4.0/.

\section{References}

1. Yoon JH, Park CH, Chung WY. Gasless endoscopic thyroidectomy via an axillary approach: experience of 30 cases. Surg Laparosc Endosc Percutan Tech 2006;16:226-31.

2. Xu JJ, Zhang LZ, Zhang QH, et al. Clinical application of the gasless unilateral axillary approach in endoscopic thyroid surgery. Zhonghua Er Bi Yan Hou Tou Jing Wai Ke Za Zhi 2020;5 5:913-20.

3. Kim MJ, Nam KH, Lee SG, et al. Yonsei Experience of 5000 Gasless Transaxillary Robotic Thyroidectomies. World J Surg 2018;42:393-401.

4. Stang MT, Yip L, Wharry L, et al. Gasless Transaxillary Endoscopic Thyroidectomy with Robotic Assistance: A High-Volume Experience in North America. Thyroid 2018;28:1655-61.

5. Barczyński M, Randolph GW, Cernea CR, et al. External branch of the superior laryngeal nerve monitoring during thyroid and parathyroid surgery: International Neural Monitoring Study Group standards guideline statement. Laryngoscope 2013;123 Suppl 4:S1-S14.

6. Zhu J, Tian W, Xu Z, et al. Expert consensus statement on parathyroid protection in thyroidectomy. Ann Transl Med 
2015;3:230.

7. Ilahi A, Muco E, Ilahi TB. Anatomy, Head and Neck, Parathyroid. Treasure Island (FL): StatPearls Publishing, 2020.

8. Li W, Wang B, Jiang ZG, et al. The role of thymus preservation in parathyroid gland function and surgical completeness after bilateral central lymph node dissection for papillary thyroid cancer: A randomized controlled study. Int J Surg 2019;65:1-6.

9. Teshima M, Otsuki N, Morita N, et al. Postoperative hypoparathyroidism after total thyroidectomy for thyroid cancer. Auris Nasus Larynx 2018;45:1233-8.

10. Wong A, Wong JCY, Pandey PU, et al. Novel techniques for intraoperative parathyroid gland identification: a comprehensive review. Expert Rev Endocrinol Metab 2020;15:439-57.

11. Randolph GW, Dralle H; International Intraoperative Monitoring Study Group, et al. Electrophysiologic recurrent laryngeal nerve monitoring during thyroid and parathyroid surgery: international standards guideline statement. Laryngoscope 2011;121 Suppl 1:S1-S16.

12. Jiang H, Shen H, Jiang D, et al. Evaluating the safety of the Harmonic Scalpel around the recurrent laryngeal nerve. ANZ J Surg 2010;80:822-6.

Cite this article as: Zhou Y, Cai Y, Sun R, Shui C, Ning Y, Jiang J, Wang W, Sheng J, Jiang Z, Tian Z, Tian W, Zheng C, Ge M, Li C. Gasless transaxillary endoscopic thyroidectomy for unilateral low-risk thyroid cancer: Li's six-step method. Gland Surg 2021;10(5):1756-1766. doi: 10.21037/gs-21-257
13. Chabrillac E, Zerdoud S, Fontaine S, et al. Multifocal recurrence on the transaxillary robotic thyroidectomy incision. Eur Ann Otorhinolaryngol Head Neck Dis 2020;137:59-60.

14. Harach HR, Cabrera JA, Williams ED. Thyroid implants after surgery and blunt trauma. Ann Diagn Pathol 2004;8:61-8.

15. Beninato T, Kleiman DA, Scognamiglio T, et al. Tract recurrence of a follicular thyroid neoplasm following transaxillary endoscopic thyroidectomy. Thyroid 2012;22:214-7.

16. Wang J, Gao L, Song C, et al. Incidence of metastases from 524 patients with papillary thyroid carcinoma in cervical lymph nodes posterior to the sternoclavicular joint (level VIa): Relevance for endoscopic thyroidectomy. Surgery 2016;159:1557-64

17. Kim EY, Lee KH, Park YL, et al. Single-Incision, Gasless, Endoscopic Trans-Axillary Total Thyroidectomy: A Feasible and Oncologic Safe Surgery in Patients with Papillary Thyroid Carcinoma. J Laparoendosc Adv Surg Tech A 2017;27:1158-64.

(English Language Editor: J. Gray) 